\title{
Stabilization Of The Spatial Double Inverted Pendulum Using Stochastic Programming Seen As a Model of Standing Postural Control
}

\author{
Xinjilefu, Vincent Hayward, and Hannah Michalska
}

\begin{abstract}
The stabilization of a double inverted pendulum moving in a three dimensional space may be considered to be a model of a human - and of other animals - postural control. Here, we show that postural control is possible by on-line minimization of the system Lagrangian. An stochastic programming procedure proves to be able to find oscillatory inputs that bring the system close to the unstable upright equilibrium position. In conclusion, our study demonstrates that steering complex mechanical systems may in certain cases be actually be simpler than expected.
\end{abstract}

\section{INTRODUCTION}

Some apparently simple systems, upon study, reveal surprinsingly complicated properties and are hard to control. Such is the case of the double inverted pendulum in the plane, see for example [1]. Conversely, complex behaviors of some complicated systems can be controlled relatively simply [2], or behave in a complex manner with no control at all [3].

We hypothesized that the maintenance of the standing posture on a small footprint, observed in many animals (such as birds, viverridae, and hominids, see Fig. 1), is such an example of complex systems which can be controlled relatively simply. Finding effective control strategies that are applicable to this type of systems would contribute to the art of humanoid design and control.

In the robotic literature, stabilization of the standing posture has received virtually no attention, presumably because it is viewed as a limiting case of walking, and hence is seen as a solved problem. For instance, if one adopts the Zero Moment Point (ZMP) control strategy, standing resembles the control of an inverted pendulum-type machine [4], [5]. A simple standing strategy is to maintain the projection of the machine's center of mass in a supporting polygon. However, the stabilization of a multiple inverted pendulum in space is an open control problem, therefore this strategy must rely on static self-stabilization with feet, which makes it non-robust. Robustifying approaches include the use of non-linear model predictive control to resist unpredictable disturbances, but these approaches have been applied in the plane only [6].

Postural stability has been studied extensively in humans, but it is tempting to think that similar control strategies exist across species. Classically, it is believed that postural stability

X. Xinjelifu and H. Michalska are with McGill University, Department of Electrical and Computer Engineering, Montréal, Canada, hannah.michalska@mcgill.ca. V. Hayward is with UPMC Univ Paris 06, UMR 7222, Institut des Systèmes Intelligents et de Robotique, France,vincent.haywardeisir.fr. Supported by Natural Sciences and Engineering Council of Canada (NSERC) and the Université Pierre et Marie Curie (UPMC). To appear in the Proceedings of the 9th IEEE-RAS International Conference on Humanoid Robots (Humanoids09), Dec. 2009. is achieved through a combination of seamlessly integrated strategies, roughly organized in a hierarchical manner to resist increasingly strong disturbances, namely, body movements involving the hip (cyclical or transient), larger movements involving the upper limbs, ankle torque production, and finally complete readjustments though steps [8], [9], [10]. This coarse picture would not be complete without mentioning the sensing aspects of standing (which we ignored in the present study). A standing individual is thought to collect information through vision [11], touch [12], proprioception [13], and vestibular inputs [14], in order to activate hundreds of muscles resulting in a formidable sensor integration and motor coordination problem.

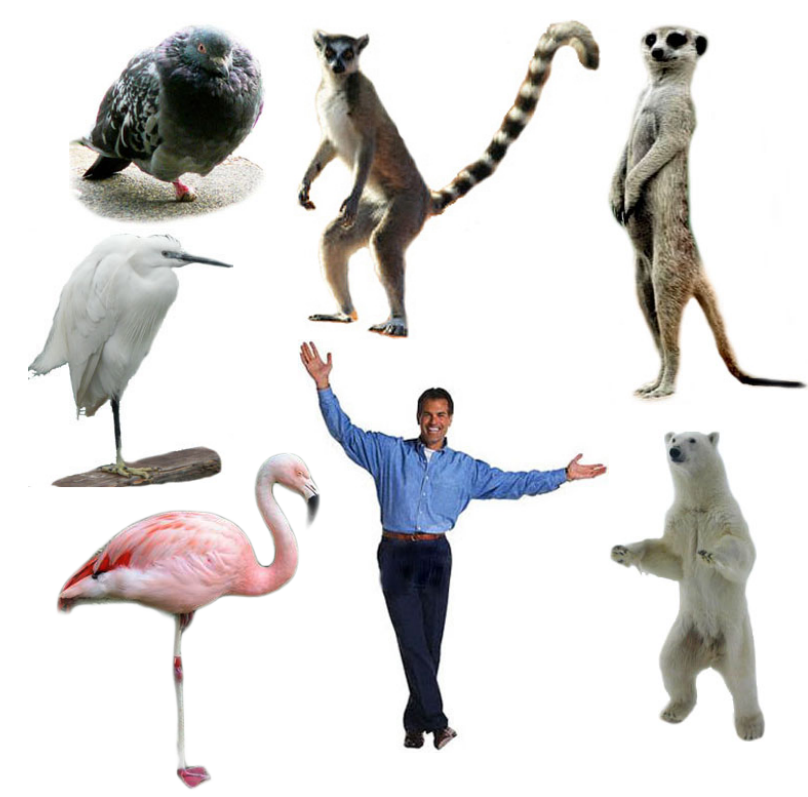

Fig. 1. Birds, viverridea, and people, among several other species, master standing behaviors on a small footprint. The poor crippled pigeon has no difficulties standing quietly on single stump; the tails of the meerkat and of the pigeon seem to play a sensing rather than a mechanical stabilization role (something analogous is well documented in humans [7].); the single-leg posture of the flamingo and of the crane is highly unstable. Examples such as these abound.

A literature search on studies of the control of the standing posture reveals literally thousands of entries, and yet, while a number of models have been proposed in the plane, both in the biological and in the robotics literature, to the knowledge the authors, no model, save one [15], consider stabilization in space of a standing animal or machine, which is the subject of this article. 


\section{A MODEL FOR THE STUDY OF STANDING IN MACHINES AND ANIMALS}

We propose that any adequate model for studying the stabilization of the standing stabilization problem should be spatial. The underlying motivation being that if the forces due to acceleration (including gravity) in a multibody system do generalize plausibly from two to three dimensions, such is not the case of centrifugal and Coriolis forces, the later being constructed out of vector products that have no equivalent in the plane. We suggest that, as far as postural stabilization is concerned, these terms, far from being mere 'additional complexities' actually are fundamental to stabilization strategies and are, of course, nonlinear in essence. Eliminating them by considering planar models (or ignoring them in spatial movements) would profundly modify the very nature of the stance stabilization problem.

The simplest multibody system which can account for these terms and yet can represent the postural control problem is the double spatial inverted pendulum which is described next.

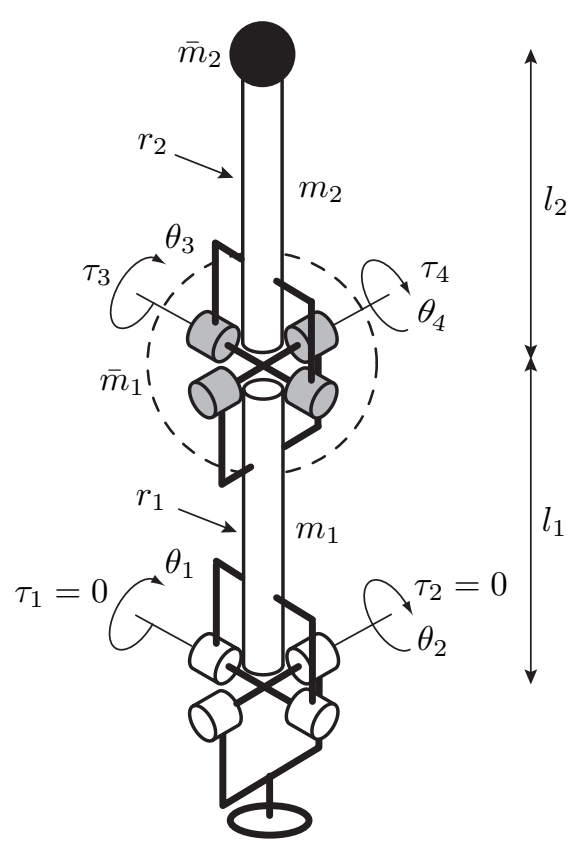

Fig. 2. The model has two main links to represent the legs and the body which are articulated at the hip and at the ankle. Addtional masses at the head at the hip are considered to provide a plausible mass distribution. Only the hip is actuated.

\section{A. A Spatial Double Inverted Pendulum Model}

This simple model captures the essential kinematic and dynamic features of standing in the upright position. Refering to Fig. 2, the model has two main rigid cylindrical links of lengths $l_{j}$, and radii $r_{j}$, with masses $m_{j}, j=1,2$, representing the upper body and legs, respectively. Additional point masses $\bar{m}_{1}$ and $\bar{m}_{2}$ are attributed to the head and to the hip, and the motion of the links is restrained by two universal joints: at the hip and at the ankle. The model is easily seen to be equivalent with a 4-revolute-joint serial manipulator, with kinematics that can be described in terms of the DenavitHartenberg (DH) parameters: $\theta_{i}, i=1, \ldots, 4$ joint angles in the DH notation. It is assumed that the balancing act is implemented by two actuating torques at the hip while the ankle is unactuated.

Taking the joints angles as generalized coordinates, $\boldsymbol{q} \triangleq$ $\left[q_{1}, \cdots, q_{4}\right]^{\top}=\left[\theta_{1}, \cdots, \theta_{4}\right]^{\top}$, in the absence of dissipation, the model is a simple Lagrangian system,

$$
L(\boldsymbol{q}, \dot{\boldsymbol{q}})=K(\boldsymbol{q}, \dot{\boldsymbol{q}})-V(\boldsymbol{q})=\frac{1}{2} \dot{\boldsymbol{q}}^{\top} M(\boldsymbol{q}) \dot{\boldsymbol{q}}-V(\boldsymbol{q}),
$$

where $\boldsymbol{q}$ is the configuration vector, $S_{2} \times S_{2} \ni \boldsymbol{q}$ is the configuration manifold, $L$ is the Lagrangian function, and $K(\boldsymbol{q}, \dot{\boldsymbol{q}})$ and $V(\boldsymbol{q})$ are the kinetic and potential energies of the system, respectively. Let $\sin q_{i}=s_{i}$ and $\cos q_{i}=c_{i}$, for $i=1, \cdots, 4$, then $M(\boldsymbol{q})$, the symmetric, positive definite inertia matrix, has entries

$$
\begin{aligned}
& M_{1,1}=\frac{1}{2} m_{1} r_{1}{ }^{2}+\frac{1}{2} m_{2} r_{2}{ }^{2}+\left(G-\frac{1}{4} m_{1} r_{1}{ }^{2}\right) c_{2}^{2} \\
& +A\left[1-\left(c_{2} s_{4}+s_{2} c_{3} c_{4}\right)^{2}\right]+2 B c_{2}\left(c_{2} c_{3} c_{4}-s_{2} s_{4}\right), \\
& M_{1,2}=s_{3} c_{4}\left[B s_{2}+A\left(c_{2} s_{4}+s_{2} c_{3} c_{4}\right)\right] \text {, } \\
& M_{1,3}=c_{4}\left[B c_{2} c_{3}+A\left(c_{2} c_{4}-s_{2} c_{3} s_{4}\right)\right]+\frac{1}{2} m_{2} r_{2}{ }^{2} c_{2} \text {, } \\
& M_{1,4}=s_{3}\left[-2 B c_{2} s_{4}+\left(A+\frac{1}{2} m_{2} r_{2}^{2}\right) s_{2}\right] \text {, } \\
& M_{2,2}=G+\frac{1}{4} m_{1} r_{1}^{2}+\frac{1}{2} m_{2} r_{2}^{2}+A\left[1-s_{3}^{2} c_{4}^{2}\right]+2 B c_{3} c_{4} \text {, } \\
& M_{2,3}=A s_{3} s_{4} c_{4} \text {, } \\
& M_{2,4}=\left(A+\frac{1}{2} m_{2} r_{2}^{2}\right) c_{3}+B c_{4}, \\
& M_{3,3}=A c_{4}^{2}+\frac{1}{2} M_{2} r_{2}^{2} \text {, } \\
& M_{3,4}=0 \text {, } \\
& M_{4,4}=A+\frac{1}{2} m_{2} r_{2}{ }^{2} .
\end{aligned}
$$

where $A, B, G, E, F$ are constants,

$$
\begin{aligned}
& A=\frac{1}{3} m_{2} l_{2}^{2}+\bar{m}_{2} l_{2}{ }^{2}-\frac{1}{4} m_{2} r_{2}{ }^{2}, \\
& B=\left(\frac{1}{2} m_{2}+\bar{m}_{2}\right) l_{1} l_{2}, \\
& G=\left(\bar{m}_{1}+\bar{m}_{2}+\frac{1}{3} m_{1}+m_{2}\right) l_{1}^{2}, \\
& E=\left(\frac{1}{2} m_{1}+\bar{m}_{1}+m_{2}+\bar{m}_{2}\right) g l_{1}, \\
& F=\left(\frac{1}{2} m_{2}+\bar{m}_{2}\right) g l_{2} .
\end{aligned}
$$

with $g$ denoting the acceleration of gravity. The potential energy is

$$
V=E c_{1} c_{2}+F\left(c_{1} c_{2} c_{3} c_{4}-s_{1} s_{3} c_{4}-c_{1} s_{2} s_{4}\right) .
$$

If $f_{i}(q): S_{2} \times S_{2} \mapsto \mathbb{R}^{4}, i=3,4$, represent the external forces applied to the system then the Euler-Lagrange equations for the system are

$$
\frac{\mathrm{d}}{\mathrm{d} t} \frac{\partial L}{\partial \dot{\boldsymbol{q}}}-\frac{\partial L}{\partial \boldsymbol{q}}=F(\boldsymbol{q}) \boldsymbol{\tau},
$$

where $\boldsymbol{\tau} \triangleq\left[\tau_{3}, \tau_{3}\right]^{\top} \in \mathbb{R}^{2}$ and $F(\boldsymbol{q})=\left[f_{3}(\boldsymbol{q}), f_{4}(\boldsymbol{q})\right]$, with $f_{1}=[0,0,1,0]^{\top}, f_{2}=[0,0,0,1]^{\top}$, denotes the matrix of external forces. Hence, for $k=1, \cdots, 4$, the system is governed by

$$
\sum_{j} m_{k j}(\boldsymbol{q}) \ddot{q}_{j}+\sum_{i, j} \Gamma_{i j}^{k}(\boldsymbol{q}) \dot{q}_{i} \dot{q}_{j}+g_{k}(\boldsymbol{q})=e_{k}^{\top} F(\boldsymbol{q}) \boldsymbol{\tau},
$$


where $e_{k}$ is the $k$-th standard basis vector in $\mathbb{R}^{4}$, and where the gravity terms and the Christoffel symbols are given by

$$
\begin{aligned}
g_{k}(\boldsymbol{q}) & =\frac{\partial}{\partial q_{k}} V(\boldsymbol{q}), \\
\Gamma_{i j}^{k}(\boldsymbol{q}) & =\frac{1}{2}\left(\frac{\partial M_{k j}(\boldsymbol{q})}{\partial q_{i}}+\frac{\partial M_{k i}(\boldsymbol{q})}{\partial q_{j}}-\frac{\partial M_{i j}(\boldsymbol{q})}{\partial q_{k}}\right)
\end{aligned}
$$

In vector form,

$$
M(\boldsymbol{q}) \ddot{\boldsymbol{q}}+\dot{\boldsymbol{q}}^{\top} Q(\boldsymbol{q}) \dot{\boldsymbol{q}}+G(\boldsymbol{q})=F(\boldsymbol{q}) \boldsymbol{\tau}
$$

where $Q$ is a matrix such that $C(\boldsymbol{q}, \dot{\boldsymbol{q}}) \dot{\boldsymbol{q}} \triangleq \dot{\boldsymbol{q}}^{\top} Q(\boldsymbol{q}) \dot{\boldsymbol{q}} \in \mathbb{R}^{4}$, the later definition being standard in the literature. The terms involving $\dot{q}_{i} \dot{q}_{i}$ represent the centrifugal forces and the terms involving $\dot{q}_{i} \dot{q}_{j}, i \neq j$, stand for Coriolis forces. Also, $G(\boldsymbol{q})=$ $\left[g_{1}(\boldsymbol{q}), \ldots, g_{4}(\boldsymbol{q})\right]^{\top}$ contains the gravity terms.

Using (21), it is then possible to show that the matrix $\frac{\mathrm{d}}{\mathrm{d} t} M(\boldsymbol{q})-2 C(\boldsymbol{q}, \dot{\boldsymbol{q}})$ is skew-symmetric. Recalling that $M(\boldsymbol{q})$ is positive definite and hence invertible, and introducing the Legendre transformation with respect to $\dot{\boldsymbol{q}}$ [16],

$$
\boldsymbol{p}=\frac{\partial L}{\partial \dot{\boldsymbol{q}}}=M(\boldsymbol{q}) \dot{\boldsymbol{q}}
$$

then allows one to rewrite the model of the system (22) in the Legendre normal form,

$$
\begin{aligned}
& \dot{\boldsymbol{q}}=M^{-1}(\boldsymbol{q}) \boldsymbol{p} \\
& \dot{\boldsymbol{p}}=-G(\boldsymbol{q})+\boldsymbol{p}^{\top}\left(M^{\top}\right)^{-1}(\boldsymbol{q}) Q(\boldsymbol{q}) M^{-1}(\boldsymbol{q}) \boldsymbol{p}+F(\boldsymbol{q}) \boldsymbol{\tau} .
\end{aligned}
$$

Stacking up $\boldsymbol{q}$ and $\boldsymbol{p}$ into $\boldsymbol{x} \triangleq[\boldsymbol{q} ; \boldsymbol{p}]$ allows one to see that the Legendre normal form of the model takes the form of a smooth nonlinear system which is affine in the control,

$$
\begin{aligned}
{\left[\begin{array}{c}
\dot{\boldsymbol{q}} \\
\dot{\boldsymbol{p}}
\end{array}\right] } & =\left[\begin{array}{c}
M^{-1}(\boldsymbol{q}) \boldsymbol{p} \\
-G(\boldsymbol{q})+\boldsymbol{p}^{\top} \tilde{Q}(\boldsymbol{q}) \boldsymbol{p}
\end{array}\right]+\left[\begin{array}{c}
0 \\
F(\boldsymbol{q})
\end{array}\right] \boldsymbol{\tau} \\
& \triangleq f(\boldsymbol{x})+g(\boldsymbol{x}) \boldsymbol{\tau}=f(\boldsymbol{x})+f_{3} \tau_{3}+f_{4} \tau_{4}
\end{aligned}
$$

where $\tilde{Q}(\boldsymbol{q}) \triangleq\left(M^{\top}\right)^{-1} Q M^{-1}(\boldsymbol{q})$ and where $f: \boldsymbol{x} \rightarrow \mathbb{R}^{8}$ is the drift vector field that is clearly related to the gravity field, and where $f_{1}, f_{2}$ are constant vector fields as defined in (18).

Although many analytical tools are available for controllability, observability analysis, and control design, for systems in the form of (26) it is prohibitively difficult, if not impossible, to use them here directly given the enormous size of the expression for the system drift. Direct calculation of the Coriolis and centrifugal forces appearing in $C(\boldsymbol{q}, \dot{\boldsymbol{q}})$ which are expressed through the Christoffel symbols (21) fill very many lines. It is apparent that an exact analysis of the structure of the controllability Lie algebra for the system is practically impossible in its present form as it requires the evaluation of repeated Lie brackets of the vector fields $f(\cdot), f_{1}, f_{2}$.

Current efforts are aimed at reducing the size of the expression through other choices of the generalized coordinates.

\section{B. Model Properties}

To further characterize the "simple" system at hand, it is worth pointing out that (22) is under-actuated with control deficiency degree two, that is, the difference between $\operatorname{rank} F(\boldsymbol{q})$ and the dimension of the configuration manifold. Also, the first two equations in (22) constitute a nonlinear motion constraint on the accelerations $\ddot{q}_{1}, \ddot{q}_{2}$ which cannot be integrated even partially, i.e., the constraints cannot be transformed into an equivalent form that contains only velocities and positions. The relation between integrability and the presence of the gravity term is discussed in [17] and [18] where sufficient conditions for integrability of second order constraints on the system accelerations are given. For a system to be integrable, the gravity terms need to be constant, but here they depend nonlinearly on the configuration variables. Non-integrability of the acceleration constraints, putting the system in the category of nonholonomic systems of order two, further signifies that the dimension of the configuration manifold is not reduced by invoking the constraints. A further implication is the lack of existence of diffeomorphic state-feedback transformations that linearize the system globally over the configuration manifold.

Notwithstanding this fact, local linearization of the system in the neighborhood of the unstable equilibrium point, which corresponds to the upright standing position, is controllable, again due to the presence of the gravity term; for the latter association, again refer to [17] and [18]. This allows one to conjecture about local controllability of the system in the neighborhood of this equilibrium and make the immediate construction of linear controllers possible, e.g., of the LQR type. Due to their inherent robustness properties, the latter also provide for asymptotic stabilization of the original model. Nevertheless, as verified by simulations, the region of attraction for this type of stabilizing feedback is unsatisfactorily small [19], [20].

The presence of the gravity term and associated drift in the Legendre normal form of the system is hence a proverbial "mixed blessing" since small time local controllability (STLC) of the system at every configuration point away from the equilibrium, a detailed analysis of the structure of controllability Lie algebra of the system is required; see [21].

\section{PREVIOUS APPROACHES TO BALANCE CONTROL}

A cornucopia of analytical techniques have been proposed to achieve semi-global or global stabilization of systems of interest. Their common thread is to generate control inputs that counteract the action of the system drift vector field. Approaches include control design constructions based on partial linearization, local transformations, triangular or chained forms, all of which apply only to systems with much simpler analytical representations, predominantly in the plane, and which are valid in a strictly restricted neighborhood of the equilibrium [22], [23], [24].

More global approaches include swing-up control combined with local linear control [15], [25], energy shaping in 
the controlled Lagrangian, controlled Hamiltonian formulations [26], [27], [28], or else interconnection and damping assignment passivity based control [29], [30]. Energy shaping and involve the solution of complex matching conditions to insure equivalence of the system Lagrangians or Hamiltonians with their controlled reduced counterparts and passivitybased approaches meet with the difficulty of the analytical construction of dissipation terms. So far, these approaches have been successfully applied to simple planar systems, or to systems of degree one of under-actuation.

The above-mentionned approaches, relying on the construction of nonlinear transformations, of matching conditions, or of suitable dissipation terms, do not seem to be applicable to our standing model due to the formidably complex expressions for the system drift vector field.

\section{StEERING THE LAGRANGIAN BY OSCILLATORY INPUTS}

Confronting the stabilization problem requires either powerful approximation/reduction of the representation of the drift vector field, or the construction of a time-varying, piecewise open-loop, feedback control akin to swing-up control. While the vector field approximation strategies are still in a theoretical fog, time-varying feedback with direct, geometrical constructions to steering, provides a firm foundation. Counteracting the effect of the drift vector field requires the generation of system motions along Lie-bracket directions that point approximately towards an increase of the potential energy of the system. In other words, we would like to search for inputs that non-instantaneously steer the system along the "good" Lie-bracket vector fields in the Lie algebraic extension of the system, see [31]. These directions cannot be evaluated exactly, but can be delivered by a "learning" procedure able to steer the system Lagrangian towards its global minimum. More precisely, if $\operatorname{TM}\left(S_{2} \times S_{2}\right)$ is the tangent bundle to the configuration manifold, the global solution to the minimization problem,

$$
\min _{(\boldsymbol{q}, \dot{\boldsymbol{q}}) \in \mathrm{TM}\left(S_{2} \times S_{2}\right)} L(\boldsymbol{q}, \dot{\boldsymbol{q}})
$$

delivers the point $\mathbf{0} \triangleq[0,0,0,0]^{\top}$ at which the system is at an unstable equilibrium. To see this, proceed by contradiction. Suppose that $\mathbf{0}$ is not the global optimum in (27). There must then exists a point $\left(\boldsymbol{q}^{*}, \dot{\boldsymbol{q}}^{*}\right)$ such that $L\left(\boldsymbol{q}^{*}, \dot{\boldsymbol{q}}^{*}\right)<L(\mathbf{0})$. But $K\left(\boldsymbol{q}^{*}, \dot{\boldsymbol{q}}^{*}\right)>K\left(\boldsymbol{q}^{*}, 0\right)$ for any $\boldsymbol{q}^{*}$ and any $\dot{\boldsymbol{q}}^{*} \neq \mathbf{0}$, and $V\left(\boldsymbol{q}^{*}\right)<V(\mathbf{0})$ for any $\boldsymbol{q}^{*} \neq 0$ by the physical meaning of the generalized coordinates and the expressions for the potential and kinetic energies of the system. Hence, $L\left(\boldsymbol{q}^{*}, \dot{\boldsymbol{q}}^{*}\right)>K(\mathbf{0}, \mathbf{0})-V(\mathbf{0})=L(\mathbf{0}, \mathbf{0})$ which contradicts the premise.

The principles for the construction of controls that steer the system along selected Lie-bracket directions are now well established; see [32], [31]. These principles permit a smooth parametrization in terms of linear combinations of complex exponentials characterized by different frequencies which, we hoped, could be found by a "learning" strategy.

\section{A. Lagrangian Minimization by Stochastic Programming}

The notion of a "good" direction will henceforth be replaced by any direction for which there exists a sinusoidal input of the form

$$
\tau_{i}=\sum_{k=1}^{N} A_{k}^{i} \sin \left(\omega_{k}^{i} t+\phi_{k}^{i}\right), \quad i=1,2,
$$

such that when the latter is applied to the system over a time horizon $[0, T]$, it brings the system to a configuration at which the value of the Lagrangian is reduced. Finding the values of $P=\left\{T, N, A_{k}^{i}, \omega_{k}^{i}, \phi_{k}^{i}\right\}, i=1,2 ; k=1, . ., N$ can be attempted by global optimization procedure aimed at the solution of (27). Although the latter optimization problem is smooth, its nature is global hence justifying stochastic programming approaches such as simulated annealing. Such a procedure is described by the pseudo code below, whererein $(\boldsymbol{q}, \dot{\boldsymbol{q}})$ is the tangent bundle, $L_{\text {min }}$ is the Lagrangian value in the upright posture, $\epsilon$ is the tolerance, Lagrangian() returns the value of Lagrangian, neighbor() randomly generates (with uniform distribution) a tentative set of parameters values in a neighborhood, and dynamics() displaces the robot given an input and initial conditions. The parameters $\alpha$ and $\beta$ determine the cooling and reheating schedule and $C$ is the temperature.

$$
\begin{aligned}
& (\boldsymbol{q}, \dot{\boldsymbol{q}}) \leftarrow\left(\boldsymbol{q}_{0}, \dot{\boldsymbol{q}}_{0}\right) \\
& L_{0} \leftarrow \text { Lagrangian }\left(\boldsymbol{q}_{0}, \dot{\boldsymbol{q}}_{0}\right) \\
& P_{\text {epoch }} \leftarrow P_{0} \\
& C \leftarrow C_{0} \\
& \text { while } L_{0}-L_{\min }>\epsilon \\
& \text { Improvement } \leftarrow \text { false } \\
& \text { while } \neg \text { Improvement and limited number of epochs } \\
& L \leftarrow \text { Lagrangian }(\boldsymbol{q}, \dot{\boldsymbol{q}}) \text { - Current Lagrangian } \\
& L_{\text {epoch }} \leftarrow L \quad \text { - Initialize epoch } \\
& C_{\text {epoch }} \leftarrow C \quad \text { - Initialize temperature } \\
& \text { for }[1, \cdots, E p o c h] \text { and not too many rejects } \\
& P_{\text {tent }} \leftarrow \text { neighbor }\left(P_{\text {epoch }}\right) \\
& \left(\boldsymbol{q}_{\text {tent }}, \dot{\boldsymbol{q}}_{\text {tent }}\right) \leftarrow \operatorname{dynamics}\left(P_{\text {tent }},(\boldsymbol{q}, \dot{\boldsymbol{q}})\right) \\
& L_{\text {tent }} \leftarrow \text { Lagrangian }\left(\boldsymbol{q}_{\text {tent }}, \dot{\boldsymbol{q}}_{\text {tent }}\right) \\
& \text { if } L_{\text {tent }}<L_{\text {epoch }} \\
& \text { or } \exp \left[-\left(\frac{L_{\text {tent }}-L_{\text {epoch }}}{C_{\text {epoch }}}\right)\right]>\operatorname{Rand}(0,1) \\
& P_{\text {epoch }} \leftarrow P_{\text {tent }} \quad-\text { Accept parameters } \\
& L_{\text {epoch }} \leftarrow L_{\text {tent }} \quad \text { - Update cost } \\
& C_{\text {epoch }} \leftarrow \alpha C_{\text {epoch }}-\text { Cool down } \\
& \text { end if } \\
& \text { end for } \\
& \text { if } L_{\text {epoch }}-L<0 \\
& \text { Improvement } \leftarrow \text { True - Improvement found } \\
& \text { end if } \\
& (\boldsymbol{q}, \dot{\boldsymbol{q}}) \leftarrow \operatorname{dynamics}\left(P_{\mathrm{epoch}},(\boldsymbol{q}, \dot{\boldsymbol{q}})\right) \\
& C \leftarrow \beta C \\
& \text { end while } \\
& \text { end while }
\end{aligned}
$$




\section{B. Simulation results}

Using the values, $m_{1}=m_{2}=\bar{m}_{1}=\bar{m}_{2}=1.0 \mathrm{~kg}$, $l_{1}=l_{2}=1.0 \mathrm{~m}, r_{1}=r_{2}=0.1 \mathrm{~m}, g=9.81 \mathrm{~m} / \mathrm{s}^{2}$, many instances of sinusoidal inputs that steered the system toward its unstable equilibrium where found by the algorithm. The examples were obtained with $\epsilon=L_{\min } / 1000, C_{0}=1.6$, with at most 20 epochs of maximum length 1000 , a maximum number of rejections of $50, \alpha=0.9, \beta=0.95$, and a frequency range limited to three decades, from 0.01 to 10.0 Hz. Since the model accounts for rigid body dynamics only, discontinuous torques were perfectly admissible and the number of sinusoids in each input was $N=3$. In the system dynamics simulations, no term was neglected.

In the first example, $\boldsymbol{q}_{0}=\left[30^{\circ}, 10^{\circ},-145^{\circ},-20^{\circ}\right]^{\top}$, $\dot{\boldsymbol{q}}_{0}=\mathbf{0}$, see Fig. 3, very far from the unstable equilibrium. The algorithm found an input with low frequency torque on $\tau_{4}$ combined with a large input on $\tau_{3}$ creating a powerful "pelvic" movement that bought all four angles to small values in a first period of $0.5 \mathrm{~s}$. Then, with a faster input, all four angles were brought to rest at almost zero, in a second period lasting $0.25 \mathrm{~s}$. The solution to this difficult acrobatic recovery was found among 70 acceptabe inputs, while 2117 were rejected. This supports the idea that "good" Lie bracket motions are not very difficult to find on average. Notice the "roller-coster" aspect of the potential curve which betrays the non-holomonic nature of the system, requiring maneuvering.

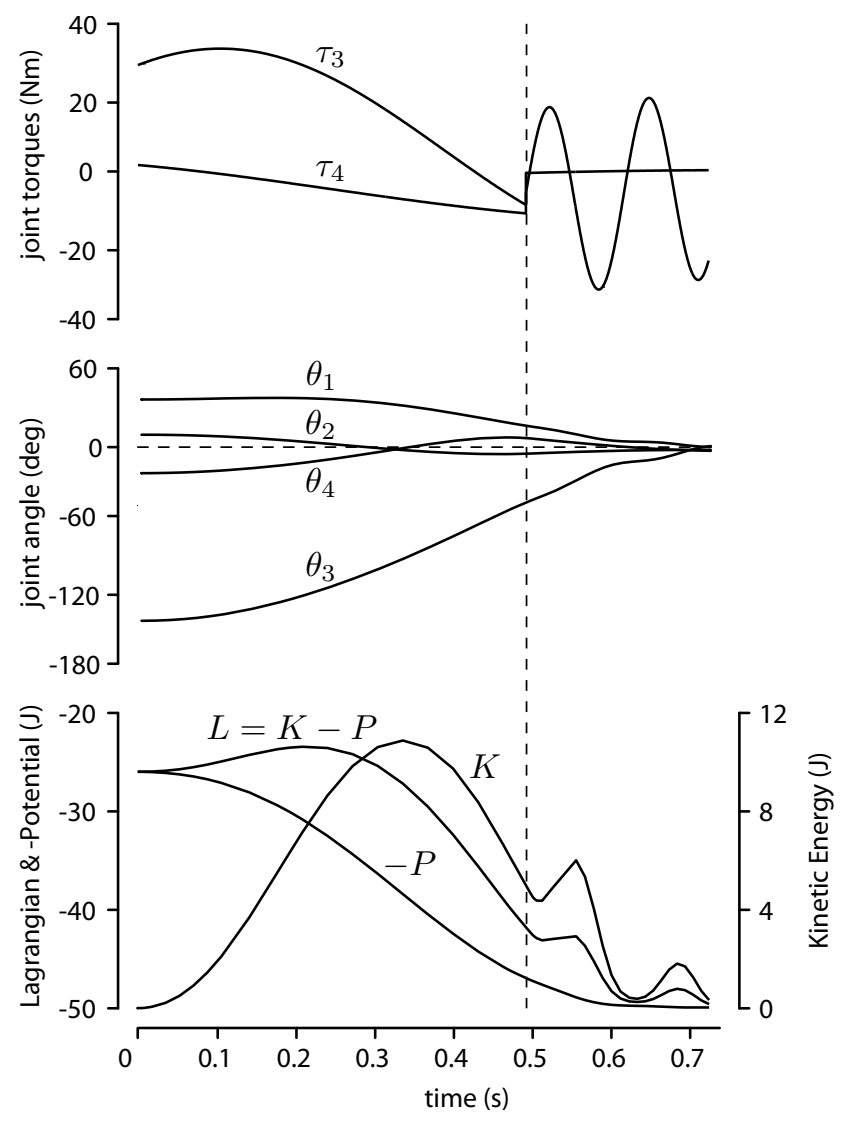

Fig. 3. Example 1. Two epochs are sufficient to bring the robot to near equilibrium from an out-of-balance poistion in two "pelvic" movements.
The second example shows a much less acrobatic recovery from initial state $\boldsymbol{q}_{0}=\left[10^{\circ}, 10^{\circ},-30^{\circ}, 30^{\circ}\right]^{\top}, \dot{q}_{0}=\mathbf{0}$. The robot is initially not far from the equilibrium but in "kinked" position of $30^{\circ}$. A rotating hip movement $\left(\theta_{3}\right.$ and $\theta_{4}$ are "mechanically $90^{\circ}$ out of phase") smoothly brings the robot to an almost straight up position in $0.2 \mathrm{~s}$. Then, a slow movement eases it to the target. Not surprinsingly, the easier nature of this maneuver is reflected by the fact that 249 good Lie bracket motions were found for 4112 rejected which means that, here, $6 \%$ of the randomly generated motions decreased the system Lagrangian.

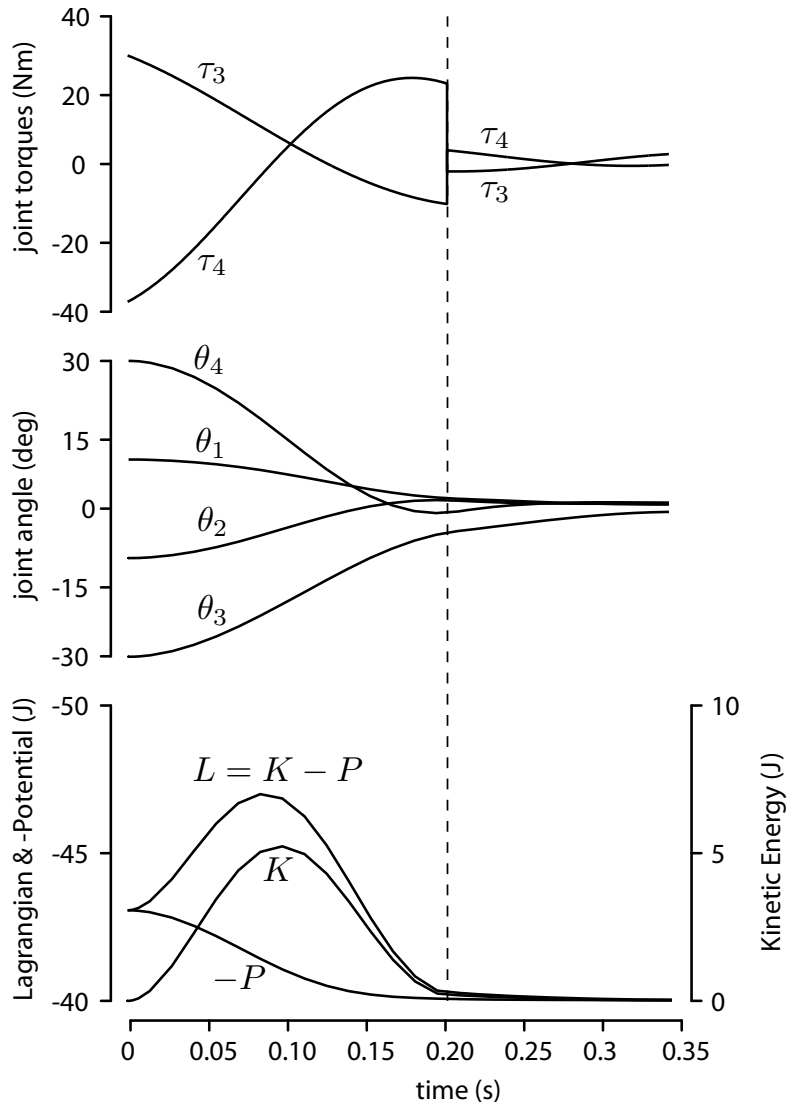

Fig. 4. Example 2. Two epochs are also sufficient to straighten the robot.

Collectivelly, the results of these experiments indicate that a relatively large proportion of all reasonable movements, on average, yield an increase of the Lagrangian, which vindicates our intuition that despite the complex nature of the control problem of the double spatial inverted pendulum, many possible inputs actually drive the Lagrangian in the "right" direction, among which a good portion counterintuitively require the system to initially move in the "wrong" direction.

\section{CONCLUSIONS AND FUTURE RESEARCH DIRECTIONS}

We considered the spatial double inverted pendulum to be a model that is well fit to study the phenomenon of the standing postural control because it is simple, yet captures the key kinematic and dynamics features of a standing animal 
or machine. We found this "simple" system to be nonlinear, under-actuated with control deficiency two, having nonintegrable constraints in acceleration which makes it nonholonomic of order two, and being associated with drift forcing small time controllability to rely on the analysis of its Lie algebra at every configuration. Together with the enormous size of the expressions that describe its dynamics, these properties put this "simple" system among the hardest to control. Yet, a standard simulated annealing procedure was able to find stabilizing inputs in most of the cases that we attempted. It is likely that this algorithm could be vastly improved with proper metaheuristics for which many candidates exist.

We are encouraged by the fact that the system turns out to be rather simple to control and that relatively numerous inputs produce good Lie bracket motions that can be found by minimizing the system Lagrangian. In its present state, the algorithm could, with certain adjustements, be implemented "online" but it is missing one crucial ingredient. The Lagrangian approach is certain to fail in the neighborhood to the upright equilibrium for the simple reason that its gradient vanishes there. Of course, the option of switching to a local linear control is available. In any case, the random search approach remains highly unsatisfactory. The reason is that, despite the fact that it seems to work in most cases, we have gained no insight as to why certain inputs succeed and why others fail, although it is possible we could work out some performance guarantees from a probabilitic view point.

For this reasons we intent to explore a number of other approaches which would be better grounded in the physics and the mathematics of this kind of systems.

\section{REFERENCES}

[1] Fliess, M., Lévine, J., Martin, P., and Rochon, P. "Flatness and defect of nonlinear systems: introductory theory and examples", International Journal of Control, Vol. 61, 1995, pp. 1327-1361.

[2] Raibert, M. H., Brown, H. B., Jr, and Chepponis, M., "Experiments in balance with a 3D one-legged hopping machine", International Journal of Robotics Research, 1984, Vol. 3, pp. 75-92.

[3] McGeer, T., "Passive bipedal running", Proceedings of the Royal Society of London. Series B, Biological Sciences, Vol. 240, No. 1297, 1990, pp. 107-134.

[4] Sugihara, T., Nakamura, Y., and Inoue, H. "Realime Humanoid Motion Generation Through ZMP Manipulation Based On Inverted Pendulum Control", Proceedings of the IEEE International Conference on Robotics and Automation, 2002, pp. 1404-1409.

[5] Kajita, S., Kanehiro, F., Kaneko, K., Fujiwara, K., Harada, K., Yokoi, K., Hirukawa, H., "Resolved momentum control: humanoid motion planning based on the linear and angular momentum,", Proceedings of the IEEE/RSJ International Conference on Intelligent Robots and Systems, Vol. 2, 2003, pp. 1644-1650.

[6] Azevedo, C., Poignet, P., and Espiau, B. "Articial locomotion control: from human to robots", Robotics and Autonomous Systems, 2004, Vol. 47, pp. 203-223.

[7] Jeka, J. J., Lackner, J. R. "The role of haptic cues from rough and slippery surfaces in human postural control", Experimental Brain Research, Vol. 103, No. 2, 1995, pp. 267-276

[8] Balasubramaniam, R. and Wing, A. M. "The dynamics of standing balance", Trends in Cognitive Sciences, vol. 6, no. 4, pp. 531-536, 2002.

[9] Bardy, B., Oullier, O., Bootsma, R., and Stoffregen, T. "Dynamics of human postural transitions", Journal of Experimental Psychology, Vol. 28, No. 3, 2002, pp. 499-514.

[10] Yang, J. F., Winter D. A, and Wells, R. P. "Postural dynamics in the standing human", Biological Cybernetics, Vol. 62, 1990, pp. 309-320.
[11] Nashner, L., Berthoz, A. "Visual contribution to rapid motor responses during postural control", Brain Research, Vol. 150, No. 2, pp. $403-$ 407.

[12] Clapp, S. and Wing, A.M. Light touch contribution to balance in normal bipedal stance. Experimental Brain Research, Vol. 125, 1999, pp. 521-524.

[13] Kavounoudias, A., Roll, A., and Roll, J. P., "Foot sole and ankle muscle inputs contribute jointly to human erect posture regulation", Journal of Physiology, Vol. 532, No. 3, 2001, pp. 869-878.

[14] Allum, J. H., Honegger, F., Schicks, H., "The influence of a bilateral peripheral vestibular deficit on postural synergies", Journal of Vestibular Research, Vol. 4, No. 1, 1994, pp. 49-70.

[15] Miyashita, N., Kishikawa, M., and Yamakita, M. "3D Motion Control of 2 links (5 D.O.F.) Underactuated Manipulator named AcroBOX", Proceedings of the 2006 American Control Conference, 2006, pp. 5614-5619.

[16] Marsden, J. E. and Ratiu, T. S., Introduction to mechanics and symmetry: A basic exposition of classical mechanical systems, Second Edition, Springer-Verlag, New York, 1999.

[17] Oriolo, G., Nakamura, Y., "Control of mechanical systems with second-order nonholonomic constraints: underactuated manipulators", Proceedings of the 30th IEEE Conference on Decision and Control, 1991, pp. 2398-2403.

[18] Wichlund, K. Y., Sørdalen, O. J., Egeland, O., "Control of vehicles with second-order nonholonomic constraints: Underactuated vehicles," Proceedings of the 3rd European Control Conference, 1995, pp. 30863091.

[19] Ayoub, O. Robotic Model of the Human Standing Posture, M.Eng Thesis, Department of Electrical and Computer Engineering, McGill University, 2005.

[20] Sood, G., Simulation and Control of a Hip Actuated Robotic Mode for the Study of Human Standing Posture, M.Eng Thesis, Department of Electrical and Computer Engineering, McGill University, 2008.

[21] Reyhanoglu, M., van der Schaft, A., McClamroch, N.H., Kolmanovsky, I., "Dynamics and control of a class of underactuated mechanical systems", IEEE Transactions on Automatic Control, Vol. 44, No. 9, 1999, pp. 1663-1671.

[22] Aneke, N. P. I., Nijmeijer, H., de Jager, A. G., "Trajectory tracking by cascaded backstepping control for a second-order nonholonomic mechanical system", in Nonlinear control in the Year 2000, SpringerVerlag, New York, 2000, pp. 35-47.

[23] Ge, S. S., Sun, Z., Lee, T. H., Spong, M. W., "Feedback linearization and stabilization of second-order nonholonomic chained systems", International Journal of Control, Vol. 74, No. 14, 2001, pp. 13831392.

[24] Xu, W. L., Ma, B. L., "Stabilization of second-order nonholonomic systems in canonical chained form", Robotics and Autonomous Systems, Vol. 34, 2001, pp. 223-233.

[25] Lai, X.-Z., Yang, S. X., Wu, M., "Singularity avoidancefor acrobots based on fuzzy-control strategy", Robotics and Autonomous Systems, Vol. 57, 2009, pp. 202-211.

[26] Bloch, A. M., Leonard, N. E., Marsden, J. E., "Controlled Lagrangians and the stabilization of mechanical systems I: the first matching theorem", IEEE Transactions on Systems and Control, Vol. 45, 2000, pp. 2253-2270.

[27] Nair, S., Leonard, N. E., "A normal form for energy shaping: application to the Furuta pendulum", Proceedings of the 41 st IEEE Conference on Decision and Control, 2002, Vol. 1, pp. 516-521.

[28] Viola, G., Ortega, R., Banavar, J. A., Acosta, J. A., Astolfi, A., "Total energy shaping control of mechanical systems: simplifying the matching equations via coordinate changes", IEEE Transactions on Systems and Control, Vol. 52. No.6, 2007, pp. 1093-1099.

[29] Ortega, R., van der Schaft, A., Maschke, B., Escobar, G., "Interconnection and damping assignment passivity-based control of port-controlled Hamiltonian systems", Automatica, Vol. 38, 2002, pp. 585-596.

[30] Acosta, J. A., Ortega, R., Astolfi, A., Mahindrakar, A. D., "Interconnection and damping assignment passivity-based control of mechanical systems with underactuation degree one", IEEE Transactions on Systems and Control, Vol. 50, No. 12, 2005, pp. 1936-1955.

[31] Sussmann, H., Liu, X., "Lie bracket extensions and averaging: the single bracket case", in Nonholomonic Motion Planning, Li, Z., Canny, J.F. (Eds.), Kluwer Academic Publishers, 1993, pp. 110-148.

[32] Murray, R. M., Sastry, S.S., "Nonholonomic motion planning: steering using sinusoids", IEEE Transactions on Automatic Control, Vol. 38, No. 5, 1993, pp. 700-716. 